\title{
Generalized rotational Hamiltonians from non-linear angular momentum algebras
}

\author{
A. Ballesteros $\left.{ }^{a}\right)$, O. Civitarese $^{b)}$, F.J. Herranz ${ }^{a}$, and M. Reboiro ${ }^{b), c)}$ \\ ${ }^{a)}$ Departamento de Física, Universidad de Burgos, Pza. Misael Bañuelos, E-09001 Burgos, Spain \\ ${ }^{b)}$ Department of Physics, University of La Plata, c.c. 67 1900, La Plata, Argentina and \\ c) Faculty of Engineering, University of Lomas de Zamora C. C. Km 2, (1836) Lavallol, Argentina
}

(Dated: January 15, 2019)

\begin{abstract}
Higgs algebras are used to construct rotational Hamiltonians. The correspondence between the spectrum of a triaxial rotor and the spectrum of a cubic Higgs algebra is demonstrated. It is shown that a suitable choice of the parameters of the polynomial algebra allows for a precise identification of rotational properties. The harmonic limit is obtained by a contraction of the algebra, leading to a linear symmetry.
\end{abstract}

PACS numbers: 21.60.-n; 21.60.Fw; 21.60.Jz; 03.65.Fd

\section{INTRODUCTION}

Mathematical aspects of the Higgs algebras have been studied intensively in the past [1, 2, 3]. Generally speaking, in the framework of non-linear algebras, the commutation relations between the generators are expressed as a linear combination of power terms of them, this is the case of the $s l^{(3)}(2, \mathbb{R})$ algebra, where the commutator of the ladder operators contains up to cubic powers of $J_{3}$. In spite of their relative involved structures, the fact that the algebras contain as limiting cases the more familiar structures of the groups associated to rotations and vibrations turns out to be a particularly appealing feature for applications to the group classification of nuclear Hamiltonians [4, 5]. Therefore, they may be useful tools to classify observables. The search for physical inspired Hamiltonians, which may display definite features about polynomial algebras is still open. Concrete applications of the formalism have been explored more recently in the context of schematic models [6], to complement previous mathematical efforts, like the studies of [7, 8, 9]. Among the already studied applications of the concept of nonlinear algebras, the results of [9] can be taken as definite motivations for our present effort.

In this work we continue with the study of applications of Higgs algebras, by constructing a Hamiltonian which reproduces the behavior of an asymmetric rotor and its vibrational limit [4]. We shall show that, while the finite dimensional representations of non-linear algebras are suitable for the description of rotational-like structures, the infinite dimensional representations of a Higgs algebra contain vibrational-like structures.

The paper is organized as follows. In Section II we present the essentials of the formalism, and introduce both the infinite- and finite-dimensional representations of $s l^{(3)}(2, \mathbb{R})$. In Section III.A, we present the basic concepts which we have developed in the construction of a triaxial rotor Hamiltonian. In Section III.B we study the vibrational limit of this Hamiltonian by contracting a particular, $s l^{(3)}(2, \mathbb{R})$, Higgs algebra. Numerical results are presented in Section IV. Conclusions are drawn in Section V.

\section{POLYNOMIAL ANGULAR MOMENTUM ALGEBRAS}

To start with we shall consider polynomial angular momentum algebras up to the third power in $J_{3}[1,3]$. The commutation relations of the $s l^{(3)}(2, \mathbb{R})$ algebra are defined by [1, 2, 3]

$$
\begin{aligned}
& {\left[J_{3}, J_{ \pm}\right]= \pm J_{ \pm},} \\
& {\left[J_{+}, J_{-}\right]=\alpha J_{3}^{3}+\beta J_{3}^{2}+\gamma J_{3}+\delta,}
\end{aligned}
$$

where the parameters $\alpha, \beta, \gamma$, and $\delta$ take arbitrary real values. The corresponding Casimir operator reads

$$
\begin{aligned}
\mathcal{C}= & J_{+} J_{-}+\frac{\alpha}{4} J_{3}^{4}+\left(\frac{\beta}{3}-\frac{\alpha}{2}\right) J_{3}^{3} \\
& +\left(\frac{\alpha}{4}-\frac{\beta}{2}+\frac{\gamma}{2}\right) J_{3}^{2}+\left(\frac{\beta}{6}-\frac{\gamma}{2}+\delta\right) J_{3} .
\end{aligned}
$$

Notice that $s l^{(3)}(2, \mathbb{R})$ comprises, as particular cases, several well-known algebras. In the linear case $(\alpha=\beta=$ $0)$ we recover the following Lie algebras: i) $\operatorname{sl}(2, \mathbb{R})$ if $\delta=0$ and $\gamma$ takes any non-zero real value; (ii) $g l(2)$ if both $\gamma$ and $\delta$ take any non-zero real value; (iii) the oscillator algebra $h_{4}$ if $\gamma=0$ and $\delta$ takes any non-zero real value; and (iv) the Poincaré algebra for $\gamma=\delta=0$ [1]. In all these cases, when a parameter (either $\gamma$ or $\delta$ ) is different from zero, it can be reduced to \pm 1 by re-scaling the Lie generators. On the other hand, the quadratic algebra $s l^{(2)}(2, \mathbb{R})$ is obtained when $\alpha=0$ and $\beta \neq 0$. In this case, the linear term $\gamma J_{3}$ can be eliminated by making use of the automorphism $J_{3} \rightarrow J_{3}-\psi$, where $\psi$ is a constant that has to be fitted. We recall that $s l^{(2)}(2, \mathbb{R})$ is, for appropriate values of the constants $\beta, \gamma$ and $\delta$, the dynamical algebra for the Dicke and Da Providencia-Schütte models (see [10] and references therein). We also remark that in the cubic case given by $\alpha \neq 0$, the same kind of automorphism allows us to eliminate the quadratic term $\beta J_{3}^{2}$. Moreover, the case with $\beta=\delta=0$ corresponds to the well-known Higgs algebra 1]. This cubic algebra was found in the study of the Kepler potential on a two-dimensional space with constant curvature and 
has motivated further developments of the representation theory of polynomial algebras [2, 3]. Finally, note that from the complementary viewpoint, the structure constants $\alpha, \beta, \gamma, \delta$ in (1) can be interpreted as deformation parameters that generate a wide class of structures by taking the Poincaré algebra (with $\alpha=\beta=\gamma=\delta=0$ ) as the origin of the space of deformations.

As far as the finite-dimensional representations of $s l^{(3)}(2, \mathbb{R})$ is concerned, these are defined on the $(2 j+1)$ dimensional basis spanned by the usual vectors $|j m\rangle$ with $j=0, \frac{1}{2}, 1, \frac{3}{2}, \ldots$ and $m=-j, \ldots, 0, \ldots, j$, like, e.g. [1]

$$
\begin{aligned}
J_{3}|j m\rangle & =\left(\frac{m}{q}+c\right)|j m\rangle, \\
J_{+}|j m\rangle & =f(m)|j m+q\rangle, \\
J_{-}|j m\rangle & =g(m)|j m-q\rangle,
\end{aligned}
$$

where $q$ is a positive integer number and $c$ is a real number. For a given $j$ the product of functions $f(m)$ and $g(m)$ fulfils

$$
\begin{aligned}
& f(m-q) g(m)-f(m) g(m+q) \\
& =\alpha\left(\frac{m}{q}+c\right)^{3}+\beta\left(\frac{m}{q}+c\right)^{2}+\gamma\left(\frac{m}{q}+c\right)+\delta,
\end{aligned}
$$

for $m=-j, \ldots, j$, and the functions $f$ and $g$ also obey the constraints

$$
\begin{aligned}
& f(j)=f(j-1)=\ldots=f(j-q+1)=0, \\
& g(-j)=g(-j+1)=\ldots=g(-j+q-1)=0 .
\end{aligned}
$$

Some other finite-dimensional representations can be found in [2, 3 ].

Infinite-dimensional representations of $s l^{(3)}(2, \mathbb{R})$ can be built on the Hilbert space of number states spanned by the set $\{|n\rangle\}$, namely:

$$
\begin{aligned}
J_{3}|n\rangle & =\left(\frac{n}{q}+c\right)|n\rangle, \\
J_{+}|n\rangle & =\mathcal{F}(n)|n+q\rangle, \\
J_{-}|n\rangle & =\mathcal{G}(n)|n-q\rangle .
\end{aligned}
$$

The functions $\mathcal{F}(n)$ and $\mathcal{G}(n)$ of Eq. (6) satisfy the conditions

$$
\begin{aligned}
& \mathcal{F}(n-q) \mathcal{G}(n)-\mathcal{F}(n) \mathcal{G}(n+q) \\
& \quad=\alpha\left(\frac{n}{q}+c\right)^{3}+\beta\left(\frac{n}{q}+c\right)^{2}+\gamma\left(\frac{n}{q}+c\right)+\delta, \\
& \mathcal{G}(0)=\mathcal{G}(1) \ldots=\mathcal{G}(q-1)=0,
\end{aligned}
$$

which can be compared with the conditions given by Eqs.(3)-(15).

The expressions appearing in (3) suggest that the generators $J_{ \pm}$play the role of ladder operators with step $q$ acting on the state $|j m\rangle$. Likewise, the results shown in equations (6) relate $J_{ \pm}$to ladder operators of a Lie algebra to power $q$ [16]. In view of the correspondence of Eqs.
(3) -(5) and Eqs. (66)-(7) we stress the fact that the structure of $s l^{(3)}(2, \mathbb{R})$ allows for a generalization of the definition of the usual angular momentum operators in the rotational limit (finite-dimensional representation) and also a generalization of the harmonic oscillator in the vibrational limit (infinite-dimensional representations). In the next section we shall advanced on these concepts.

\section{GENERALISED HAMILTONIANS.}

If $s l^{(3)}(2, \mathbb{R})$ is interpreted as a dynamical algebra, then the most general Hamiltonian based on such non-linear symmetry takes the form

$$
\mathcal{H}=\sum_{i=0}^{4} C_{i} J_{3}^{i}+C_{+} J_{+}+C_{-} J_{-},
$$

where the coefficients $C_{i}$ as well as $C_{ \pm}$are arbitrary real numbers.

In order to obtain explicit models, let us introduce the following finite-dimensional representation (3)-(4) with $q=2$. The functions $f(m)$ and $g(m)$ are, for this case

$$
\begin{aligned}
& f(m)=a \sqrt{(j+m+1)(j+m+2)(j-m)(j-m-1)}, \\
& g(m)=a \sqrt{(j+m)(j+m-1)(j-m+1)(j-m+2)},
\end{aligned}
$$

where $a$ is a real constant. Notice that these functions resemble the eigenvalues of the square of the linear $\operatorname{sl}(2, \mathbb{R})$ ladder operators. Next, according to Eq. (4), the deformation parameters $\alpha, \beta, \gamma$, and $\delta$ in (1) turn out to be

$$
\begin{aligned}
& \alpha=-64 a^{2}, \quad \beta=192 a^{2} c, \\
& \gamma=-8 a^{2}\left(1+24 c^{2}-2 j(j+1)\right), \\
& \delta=8 a^{2} c\left(1+8 c^{2}-2 j(j+1)\right) .
\end{aligned}
$$

In this representation, the eigenvalue of the Casimir operator (2) reads

$$
\begin{aligned}
\langle j m|\mathcal{C}| j m\rangle=a^{2} & \left(j(j+1)-2 c-4 c^{2}\right) \\
& \times\left(j(j+1)-2-6 c-4 c^{2}\right) .
\end{aligned}
$$

The non-vanishing matrix elements of the Hamiltonian (8) are given by

$$
\begin{aligned}
& \langle j m|\mathcal{H}| j m\rangle=\sum_{i=0}^{4} C_{i}\left(\frac{m}{2}+c\right)^{i} \\
& \langle j m+2|\mathcal{H}| j m\rangle=C_{+} f(m), \\
& \langle j m-2|\mathcal{H}| j m\rangle=C_{-} g(m) .
\end{aligned}
$$

The above expressions demonstrate that finitedimensional representations of non-symmetric algebras 
are, therefore, suitable for the treatment of rotationallike structures. This property is a result of the explicit dependence of the functions $f$ and $g$ upon $j$, which is acting as the effective level of the representation since $f(m)$ and $g(m)$ vanish if $m=j, j-1$ and $m=-j,-j+1$, respectively.

Similarly, for the case of infinite-dimensional representations one has

$$
\begin{aligned}
& \mathcal{F}(n)=b \sqrt{(n+1)(n+2)}, \\
& \mathcal{G}(n)=b \sqrt{n(n-1)},
\end{aligned}
$$

provided that $q=2, b$ is a real constant, and the deformation parameters are

$$
\alpha=\beta=0, \quad \gamma=-8 b^{2}, \quad \delta=2 b^{2}(4 c-1) .
$$

Hence the eigenvalue of the Casimir operator (2) is

$$
\langle n|\mathcal{C}| n\rangle=2 b^{2} c(1+2 c) .
$$

For this case the non-vanishing matrix elements of the Hamiltonian (8) are

$$
\begin{aligned}
& \langle n|\mathcal{H}| n\rangle=\sum_{i=0}^{4} C_{i}\left(\frac{n}{2}+c\right)^{i}, \\
& \langle n+2|\mathcal{H}| n\rangle=C_{+} \mathcal{F}(n), \\
& \langle n-2|\mathcal{H}| n\rangle=C_{-} \mathcal{G}(n),
\end{aligned}
$$

to be compared with (12). Thus, infinite-dimensional representations of non-linear algebras exhibit vibrationallike structures, under a proper choice of parameters, based on the explicit dependence of $\mathcal{F}$ and $\mathcal{G}$ upon $n$.

\section{A. Triaxial rotor}

The results of the previous subsection illustrate rather explicitly the scope of the formal connection we would like to establish between finite (infinite) representations of a polynomial algebra and the generators of angular momentum (vibrational quanta) and the corresponding Hamiltonians.

Let us start with the case of a triaxial rotor [4], whose Hamiltonian is written

$$
H_{\mathrm{rot}}=\sum_{i=1}^{3} A_{i} I_{i}^{2}
$$

where

$$
A_{i}=\frac{1}{2 \mathcal{I}_{i}} .
$$

The operators $I_{i}$ are the components of the angular momentum along the principal axes, and $\mathcal{I}_{i}$ are the components of the tensor of inertia. The generators $I_{i}$, of the intrinsic angular momentum components respect to the body-fixed frame, satisfy the commutation relations $\left[I_{i}, I_{j}\right]=-\mathrm{i} \varepsilon_{i j k} I_{k}$. We define the angular momentum rising and lowering ladder operators

$$
I_{ \pm}=I_{1} \pm \mathrm{i} I_{2}
$$

which obey the commutation rules

$$
\left[I_{3}, I_{ \pm}\right]=\mp I_{ \pm}, \quad\left[I_{+}, I_{-}\right]=-2 I_{3}
$$

The Casimir invariant is given by

$$
\mathbf{I}^{2}=\sum_{i=1}^{3} I_{i}^{2}=I_{3}^{2}+\frac{1}{2}\left(I_{+} I_{-}+I_{-} I_{+}\right) .
$$

In this way the Hamiltonian (17) can be written as

$$
\begin{aligned}
H= & \frac{1}{2}\left(A_{1}+A_{2}\right) \mathbf{I}^{2}+\frac{1}{4}\left(A_{1}-A_{2}\right)\left(I_{+}^{2}+I_{-}^{2}\right) \\
& +\frac{1}{2}\left(A_{1}-A_{2}\right) \kappa I_{3}^{2},
\end{aligned}
$$

where

$$
\kappa=\frac{2 A_{3}-A_{1}-A_{2}}{A_{1}-A_{2}},
$$

is the factor which measures the asymmetry of the rotor.

In the following, we shall show that a correspondence between the triaxial rotor case and a cubic Higgs algebra can indeed be established by a proper choice of the parameters of the non-linear symmetry.

Firstly, we adopt the following choice of parameters, in Eq.(2),

$$
\alpha=-\frac{4}{I^{2}}, \quad \beta=0, \quad \gamma=\frac{1}{2 I^{2}}\left(2 I^{2}+2 I-1\right), \quad \delta=0,
$$

for the non-linear symmetry, and taking $q=2, c=0$, and $a=\frac{1}{4 I}$ in Eq. (4) one obtains

$$
\begin{aligned}
& f(M)=a \sqrt{(J+M+1)(J+M+2)(J-M)(J-M-1)} \\
& g(M)=a \sqrt{(J+M)(J+M-1)(J-M+1)(J-M+2)}
\end{aligned}
$$

where $J=I$ and $M=M_{I}$, being $I$ the total angular momentum and $M_{I}$ its projection. The operators $J_{ \pm}$ and $J_{3}$ are written

$$
J_{ \pm}=\frac{1}{4 I} I_{ \pm}^{2}, \quad J_{3}=-\frac{1}{2} I_{3} .
$$

The corresponding commutation relations are

$$
\begin{aligned}
{\left[J_{3}, J_{ \pm}\right] } & = \pm J_{ \pm} \\
{\left[J_{+}, J_{-}\right] } & =\frac{1}{2 I^{2}}\left(2 I^{2}+2 I-1\right) J_{3}-\frac{4}{I^{2}} J_{3}^{3} .
\end{aligned}
$$


Secondly, the Hamiltonian of Eq. (22) is expressed in terms of the non-linear symmetry as

$$
\begin{aligned}
\mathcal{H}= & \frac{1}{2}\left(A_{1}+A_{2}\right) J(J+1)+J\left(A_{1}-A_{2}\right)\left(J_{+}+J_{-}\right) \\
& +2\left(A_{1}-A_{2}\right) \kappa J_{3}^{2} .
\end{aligned}
$$

The matrix elements of the Hamiltonian of Eq. (28) are given by

$$
\begin{aligned}
& \langle\mathrm{J} \mathrm{M}|\mathcal{H}| \mathrm{JM}\rangle=\frac{1}{2}\left(A_{1}+A_{2}\right) J(J+1)+\frac{1}{2}\left(A_{1}-A_{2}\right) \kappa M^{2}, \\
& \langle\mathrm{~J} \mathrm{M}+2|\mathcal{H}| \mathrm{JM}\rangle=J\left(A_{1}-A_{2}\right) f(M), \\
& \langle\mathrm{J} \mathrm{M}-2|\mathcal{H}| \mathrm{JM}\rangle=J\left(A_{1}-A_{2}\right) g(M) .
\end{aligned}
$$

Both Hamiltonians, the one of Eq. (22) and the one of Eq.(28), have the same matrix elements, and in consequence the same spectrum and the same eigenfunctions.

The above example shows that the standard angular momentum operators of the triaxial rotor can be understood as the mapped version of the generators of a particular non-linear symmetry. That is to say that the triaxial rotor is a particular case of a finite-dimensional representation of $s l^{(3)}(2, \mathbb{R})$.

\section{B. Harmonic limit of the triaxial rotor.}

We shall now illustrate the use of the concept of contraction of a Higgs algebra, in connection with the structure of $H$, of Eq.(22), in the case of large angular momentum $I$. In the standard representation of angular momentum 11], the Hamiltonian of equation (22) can be analyzed by making use of boson mapping techniques [12].

We shall use the well known Holstein-Primakoff boson mapping to get the images of the operators of Eq. (20),

$$
\begin{aligned}
I_{+} & =\zeta^{\dagger} \sqrt{2 I-\zeta^{\dagger} \zeta} \\
I_{-} & =\sqrt{2 I-\zeta^{\dagger} \zeta} \zeta \\
I_{3} & =I-\zeta^{\dagger} \zeta .
\end{aligned}
$$

where $\zeta^{\dagger}(\zeta)$ are boson creation (annihilation) operators. In the large angular momentum limit, $I>>1$, the Hamiltonian of Eq. (22) reads

$$
\begin{aligned}
H & =H_{00}+H_{11}+H_{20}+H_{n n}+H^{\prime}, \\
H_{00} & =A_{3} I(I+1)-\frac{1}{2}\left(A_{1}-A_{2}\right) \kappa I, \\
H_{11} & =-\frac{1}{2}\left(A_{1}-A_{2}\right) \kappa(2 I-1) \zeta^{\dagger} \zeta, \\
H_{20} & =\frac{1}{2}\left(A_{1}-A_{2}\right) I\left(\zeta^{\dagger 2}+\zeta^{2}\right), \\
H_{n n} & =\frac{1}{2}\left(A_{1}-A_{2}\right) \kappa\left(\zeta^{\dagger} \zeta\right)^{2}, \\
H^{\prime} & =-\frac{1}{8 I}\left(A_{1}-A_{2}\right)\left(\zeta^{\dagger 3} \zeta+\zeta^{\dagger} \zeta^{3}\right) .
\end{aligned}
$$

The terms of $H$ of Eq. (31) are classified in powers of $I$, and by the corresponding powers of the boson creation and annihilation operators $\zeta^{\dagger}$ and $\zeta$

$$
\sum_{k n} a_{k n}(I) \zeta^{\dagger k} \zeta^{n}
$$

The leading order terms, $H_{\mathrm{RPA}}=H_{00}+H_{11}+H_{20}$, can be diagonalized by adopting the Random Phase Approximation (RPA) formalism [4, 12]. This is achieved by introducing the RPA boson operator

$$
\Gamma^{\dagger}=X \zeta^{\dagger}-Y \zeta,
$$

and by solving the equation of motion

$$
\left[H_{\mathrm{RPA}}, \Gamma^{\dagger}\right]=\omega \Gamma^{\dagger} \text {. }
$$

Details of the procedure, which is rather well known, can found in textbooks [4, 13]. In this approximation $H_{\mathrm{RPA}}$ takes the form

$$
H_{\mathrm{RPA}}=A_{3} I(I+1)+\omega\left(\Gamma^{\dagger} \Gamma+\frac{1}{2}\right)
$$

with

$$
\omega=2 I \sqrt{\left(A_{3}-A_{1}\right)\left(A_{3}-A_{2}\right)} .
$$

The expression for the RPA eigenfrequency $\omega$ is obtained by expressing $H_{\mathrm{RPA}}$ and $\Gamma^{\dagger}$ in terms of the operators $\zeta^{\dagger}$ and $\zeta$ and by equaling terms after performing the commutation (34).

The remaining terms $H_{n n}$ and $H^{\prime}$ of the Hamiltonian of Eq. (31), can then be transformed to the RPA boson basis $\left(\Gamma^{\dagger}, \Gamma\right)$. By keeping terms up to $\left(\Gamma^{\dagger} \Gamma\right)^{2}$ and neglecting negative powers of the angular momentum in the coefficients $a_{k n}(I)$, one gets

$$
\begin{aligned}
H \approx A_{3} I(I+1) & +\left(\omega+\frac{1}{4 \kappa}\left(A_{1}-A_{2}\right)\right)\left(\Gamma^{\dagger} \Gamma+\frac{1}{2}\right), \\
& +\frac{1}{2}\left(A_{1}-A_{2}\right) \kappa\left(\Gamma^{\dagger} \Gamma\right)^{2} .
\end{aligned}
$$

Proceeding now in a manner analogous to the previous subsection, we shall show that this Hamiltonian is obtained as a particular case of the $s l^{(3)}(2, \mathbb{R})$ algebra. As we shall demonstrate, the harmonic limit of the triaxial rotor can alternatively be obtained through a contraction procedure and next embedded, as a particular case, within the $s l^{(3)}(2, \mathbb{R})$ structure together with some infinite-dimensional representations. In what follows we describe explicitly this procedure and, furthermore, we shall show that this is equivalent to the RPA approximation. It means that, at leading order, both constructions lead to the same results. 
The contraction of the Lie algebra (20) to the harmonic oscillator algebra $h_{4}$ is obtained by introducing the new generators defined by [14]:

$$
\hat{I}_{ \pm}=\varepsilon I_{ \pm}, \quad \hat{I}_{3}=-I_{3}+\frac{1}{2 \varepsilon^{2}},
$$

where $\varepsilon$ is the contraction parameter and $\mathbf{1}$ is the identity operator, which obviously commutes with $I_{ \pm}$and $I_{3}$. By computing the new Lie brackets and next applying the limit $\varepsilon \rightarrow 0$ we find the defining relations for $h_{4}$ :

$$
\left[\hat{I}_{3}, \hat{I}_{ \pm}\right]= \pm \hat{I}_{ \pm}, \quad\left[\hat{I}_{-}, \hat{I}_{+}\right]=\mathbf{1}, \quad[\mathbf{1}, \cdot]=0 .
$$

Since the eigenvalue of $\mathbf{1}$ is always 1 in all the representations here considered, hereafter we always fix $\mathbf{1} \equiv 1$. In this respect, notice that, in fact, such an identity operator should also be introduced in the commutation relations of $s l^{(3)}(2, \mathbb{R})$ (1) multiplying the parameter $\delta$, but due to the above reason this is not usually written explicitly in the literature [1, 2, 3]].

The contracted Casimir $\hat{\mathbf{I}}^{2}$ comes from (21) as the limit

$$
\hat{\mathbf{I}}^{2}=\lim _{\varepsilon \rightarrow 0} \varepsilon^{2}\left(\mathbf{I}^{2}-\frac{1}{4 \varepsilon^{4}}\right)=-\hat{I}_{3}+\frac{1}{2}\left(\hat{I}_{+} \hat{I}_{-}+\hat{I}_{-} \hat{I}_{+}\right) .
$$

The contraction of the rotor Hamiltonian (22) follows as

$$
\begin{aligned}
\hat{H}= & \lim _{\varepsilon \rightarrow 0} \varepsilon^{2}\left(H-\frac{A_{3}}{4 \varepsilon^{4}}\right) \\
= & \frac{1}{2}\left(A_{1}+A_{2}\right) \hat{\mathbf{I}}^{2}+\frac{1}{4}\left(A_{1}-A_{2}\right)\left(\hat{I}_{+}^{2}+\hat{I}_{-}^{2}\right) \\
& \quad-\frac{1}{2}\left(A_{1}-A_{2}\right) \kappa \hat{I}_{3},
\end{aligned}
$$

where $\kappa$ is the same of Eq. (23). Now all of these results can be introduced in $s l^{(3)}(2, \mathbb{R})$ by taking the parameters (14) with $c=0$,

$$
\alpha=\beta=0, \quad \gamma=-8 b^{2}, \quad \delta=-2 b^{2},
$$

which implies that the eigenvalue of $\hat{\mathbf{I}}^{2}$ is $1 / 2$. The generators of $s l^{(3)}(2, \mathbb{R})$ are then chosen as

$$
J_{ \pm}=b \hat{I}_{ \pm}^{2}, \quad J_{3}=\frac{1}{2} \hat{I}_{3},
$$

and fulfil the commutation relations

$$
\left[J_{3}, J_{ \pm}\right]= \pm J_{ \pm}, \quad\left[J_{+}, J_{-}\right]=-8 b^{2} J_{3}-4 b^{2} \hat{\mathbf{I}}^{2} .
$$

Hence the harmonic limit of the rotor Hamiltonian arises as the $s l^{(3)}(2, \mathbb{R})$-system given by

$$
\begin{aligned}
\mathcal{H}= & \frac{1}{2}\left(A_{1}+A_{2}\right) \hat{\mathbf{I}}^{2}+\frac{1}{4 b}\left(A_{1}-A_{2}\right)\left(J_{+}+J_{-}\right) \\
& -\left(A_{1}-A_{2}\right) \kappa J_{3},
\end{aligned}
$$

which compares with the Hamiltonian of Eq.(28) up to the term proportional to $J_{3}$, due to the contraction (38). Except for an additive constant, Eq.(45) coincides with the terms of Eq.(31) labelled as $H_{\mathrm{RPA}}$.

\section{RESULTS AND DISCUSSIONS}

In this section we shall present and discuss the results of our calculations. We shall begin with the analysis of the rotational regime by calculating the eigenvalues of the Hamiltonian of Eq. (28) and then we shall proceed by showing the results corresponding to the eigenvalues of the Hamiltonian of Eq. (45). Since we have already presented the algebraic details in the previous sections II and III, we shall focus on the numerical results.

The spectrum of a triaxial rotor has remarkable features [4] like the breaking of degeneracies and the reordering of levels in degenerate pairs. The transition between both regimes is governed by the asymmetry parameter $\kappa$. These features are exhibited, too, by the spectrum of eigenvalues of the Hamiltonian (28). Figure 1 shows the spectrum as a function of the asymmetry $\kappa$ and for a fixed angular momentum, $I=10$. Figure 2 shows the low energy region of the spectrum for $I=4$. As it can be seen from both figures, the just mentioned features emerge clearly. It means that the choice (24) of the non-linear algebra $s l^{(3)}(2, \mathbb{R})$ leads to the triaxial rotor. This is an expected result, because using the algebraic procedure of Section III we have shown that the matrix elements of both Hamiltonians do indeed coincide. Figure 3 shows the eigenvalues of the Hamiltonian of Eq. (45) as a function of the asymmetry $\kappa$. The spectrum is clearly harmonic for a fixed value of $\kappa$. The dependence of the frequency $\omega$ upon $\kappa$ and angular momentum $I$ is a unique feature of the large angular momentum limit of the triaxial rotor. The domains $|\kappa|<1$, of the rotor, and $|\kappa|>1$, of the vibrator are not connected.

\section{CONCLUSIONS}

In this work we have studied the correspondence between a polynomial algebra, of the cubic Higgs-type, and the standard angular momentum and harmonic oscillator algebras. Particularly, we have shown that: (i) by a suitable choice of the parameters entering the definition of the cubic algebra, one may obtain the triaxial rotor, and, (ii) in the limit of large angular momentum, the vibrational structure emerges as a contraction of the algebra.

We think that these results suggest the existence of direct physical realizations of the Higgs algebra for the case of Hamiltonians describing nuclear vibrations and rotations, as it was found for the case of classical Hamiltonians, e.g. the study of the Kepler potential on a twodimensional curved space.

For the specific application to nuclear structure problems, the potential of non-linear algebras in dealing with the construction of Hamiltonians becomes evident. The use of non-linear algebras, like the $s l^{(3)}(2, \mathbb{R})$ or even algebras with a larger number of generators, may shead light on more involved structures lying in between the rotational and vibrational extremes. This may be the 
case of the newly discovered symmetry reported in [15]. Work is in progress concerning this point.

\section{Acknowledgements}

This work was partially supported by the CONICET (Argentina, Project PIP-02037), by the Ministerio de
Educación y Ciencia (Spain, Project FIS2004-07913), and by the Junta de Castilla y León (Spain, Project VA013C05). A.B. and F.J.H. acknowledge the hospitality received at the Department of Physics of the University of La Plata, Argentina.
[1] P. W. Higgs, J. Phys. A 12, 309 (1979).

[2] N. Debergh, J. Phys. A 33, 7109 (2000).

[3] N. Debergh, J. Ndimubandi, and B. Van den Bossche, Mod. Phys. Lett. A 18, 1013 (2003).

[4] A. Bohr and B. Mottelson, Nuclear Structure vol. 2 Benjamin, Reading, MA, 1975)

[5] F. Iachello and A. Arima, The interacting boson model. Cambridge Monographs on Mathematical Physics. Cambridge (2006).

[6] N. Debergh and Fl. Stancu, J. Phys. A 34, 3265 (2001).

[7] V. S. Kumar, B. A. Bambah and R. Jagannathan, Mod. Phys. Lett. A 17, 1559 (2002).

[8] D. Ruan, Phys. Lett. A 319, 122 (2003).

[9] N. Debergh and B. Van den Bossche, Ann. Phys. 308, 605 (2003).

[10] A. Ballesteros, O. Civitarese, F. J. Herranz and M. Reboiro, Theor. Math. Phys. 137, 1495 (2003).

[11] D. M.Brink and G. R. Satchler, Angular Momentum. Oxford Library of Physical Sciences Series, Oxford, 1994

[12] A. Klein and E. R. Marshalek, Rev. of Modern Phys. 63, 375(1991).

[13] P. Ring and P. Shuck, The nuclear many-body problem. (Springer Verlag, N.Y. 1980)

[14] A. Ballesteros, F. J. Herranz and P. Parashar, J. Phys. A 32, 2369 (1999)

[15] E.A. McCutchan and R. F. Casten, Phys. Rev. C 74, 057302(2006); E.A. McCutchan, N. V. Zamfir and R. F. Casten, Phys. Rev. C 71, 034309 (2005); E.A. McCutchan et al. , Phys. Rev. C 71, 024309(2006).

[16] Generally speaking a boson-like of the form $J_{+} \propto a^{\dagger^{q}}$, and $J_{-} \propto a^{q}$.

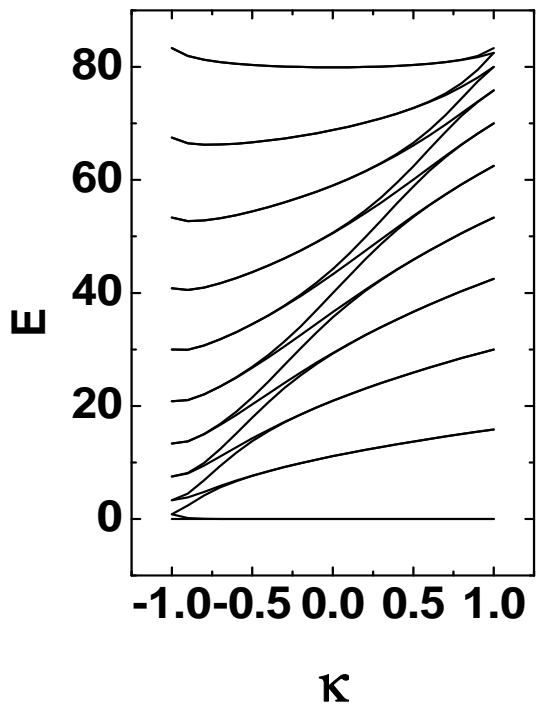

FIG. 1: Eigenvalues of the Hamiltonian of Eq. (28), as a function of the asymmetry $\kappa$. The values are shown in arbitrary units. The parameters used in the calculations are: $I=10$, $A_{1}=1.66$, and $A_{2}=0.83$. The results coincide with the ones of a triaxial rotor [4] 


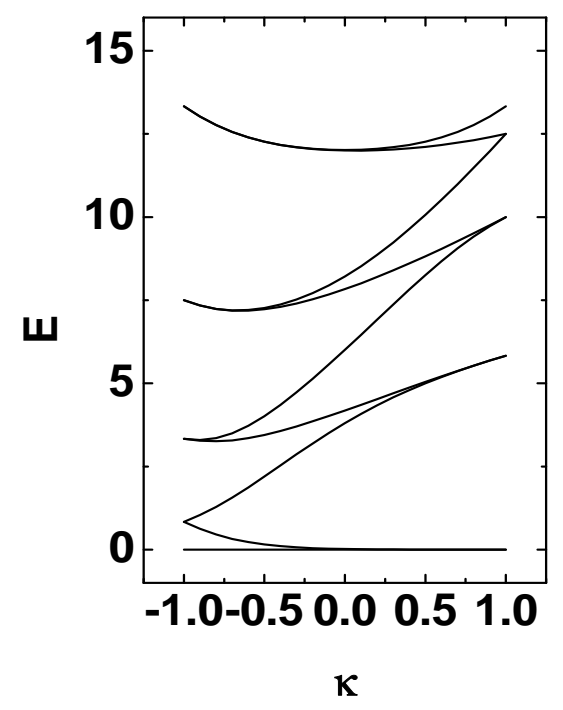

FIG. 2: Idem as Figure 1, for $I=4$.

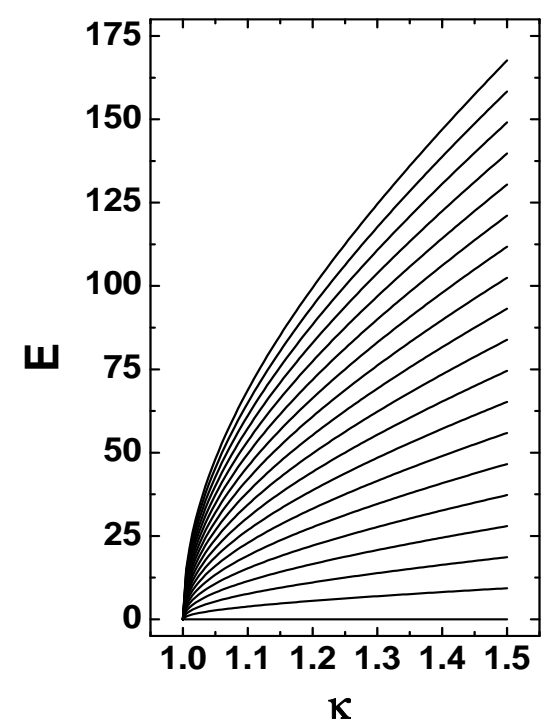

FIG. 3: Eigenvalues of the Hamiltonian of Eq.45, as a function of the asymmetry $\kappa$. The parameters are the same given in the captions to Figure 1. In curves are shown the results of the harmonic limit of Eq. (28) obtained by contracting the $s l^{(3)}(2, \mathbb{R})$ algebra. 